\title{
Understanding possible electromagnetic counterparts to loud gravitational wave events: Binary black hole effects on electromagnetic fields
}

\author{
Carlos Palenzuela ${ }^{1,2}$, Luis Lehner ${ }^{3,4,5}$ and Shin Yoshida ${ }^{6}$ \\ ${ }^{1}$ Canadian Institute for Theoretical Astrophysics (CITA), Toronto, Canada \\ ${ }^{2}$ Max-Planck-Institut für Gravitationsphysik, Albert-Einstein-Institut, Golm, Germany \\ ${ }^{3}$ Perimeter Institute for Theoretical Physics, Waterloo, Ontario N2L 2Y5, Canada \\ ${ }^{4}$ Department of Physics, University of Guelph, Guelph, Ontario N1G 2W1, Canada, \\ ${ }^{5}$ Canadian Institute For Advanced Research (CIFAR), Cosmology and Gravity Program, Canada \\ ${ }^{6}$ Department of Physics and Astronomy, University of Tokyo, Tokyo, Japan
}

(Dated: October 29, 2018)

\begin{abstract}
In addition to producing loud gravitational waves (GW), the dynamics of a binary black hole system could induce emission of electromagnetic (EM) radiation by affecting the behavior of plasmas and electromagnetic fields in their vicinity. We here study how the electromagnetic fields are affected by a pair of orbiting black holes through the merger. In particular, we show how the binary's dynamics induce a variability in possible electromagnetically induced emissions as well as an enhancement of electromagnetic fields during the late-merge and merger epochs. These time dependent features will likely leave their imprint in processes generating detectable emissions and can be exploited in the detection of electromagnetic counterparts of gravitational waves.
\end{abstract}

\section{INTRODUCTION}

The promise of detecting and analysing compact systems with both gravitational and electromagnetic waves stands out as one of the most exciting prospects in the coming decades. As already pointed out in a number of works (e.g. 1, 2]), most astrophysical systems which produce strong gravitational waves likely emit copiously in the electromagnetic band. Indeed the strong, and possibly highly dynamical, gravitational fields around compact objects affect the dynamics of plasmas and matter which in turn induce different emission mechanisms.

One example of such a system is a black hole surrounded by an accretion disk. Strong emission from these systems is understood as the result of radiative processes within jets powered by the extraction of rotational and binding energy. While the latter is qualitatively understood in terms of Newtonian-rooted arguments based on the potential of the central object, the former relies on extracting energy from a rotating black hole in the most efficient energy convertion process we know of.

The pioneering works of Penrose [3] and Blandford and Znajek [4], together with a large body of subsequent work, has provided a basic understanding of possible mechanisms to explain highly energetic emissions from single black hole systems interacting with surrounding plasmas (e.g. 5]). The interaction of electromagnetic field lines with the strong gravitational field of a rotating black hole is the fundamental component of these mechanisms to explain the acceleration of particles that traverse the black hole's ergosphere. This scenario of a pseudostationary, single black hole interacting with an accretion disk is reasonably well understood, and it is employed to explain energetic phenomena such as gamma ray bursts, AGNs, quasars, blazars, etc. However, a highly dynamical stage may occur prior to such a pseudo-stationary regime which could give rise to strong emissions. In the context of galaxy mergers, such a stage would naturally occur as individual black holes in each galaxy eventually collide in the galaxy resulting from the merger [6].

Gravitational waves from such collision would be detectable by the Laser Interferometric Space Antenna (LISA) and, as pointed out in e.g. [7, 8], the late orbitting and merger phases would take place within a circumbinary disk and possibly even interacting with some residual plasma inside the orbiting black holes [9].

Possible emissions from these systems are only understood at late times, when the the pseudo-stationary picture mentioned above is applicable. However the intermediate regime has only recently been approached by a few works [9, 10, 11] and in all cases with important simplifications introduced to track the system. In this work, as a follow up of [10], we concentrate on understanding the dynamics of possible electromagnetic fields anchored in the circumbinary disk in the presence of the merging black holes. In particular we examine the field configuration, possible energy enhancement and time variability of these fields as the merger take place and point out possible process that could give rise to a signal around the merger time. To this end, we consider the EinsteinMaxwell system in a setup that describes a pair of black holes close to the merger epoch, study the electromagnetic field behavior and compare to the single black hole case. While we do not consider a plasma in our current study, our analysis helps to understand the possible behavior in its presence and lay the foundations to future work in this direction.

This work is organized as follows, in Section II, we briefly review our formulation and numerical implementation of the problem. Section III describes the physical set up to study single and binary black hole configurations. Section IV discusses the results obtained for both scenarios considered and highlight main features which could induce emission with particular patterns. We conclude with section $\mathrm{V}$ which offers some final considerations and discussions. 


\section{FORMULATION AND NUMERICAL APPROACH}

We solve the coupled Einstein-Maxwell system to model the black hole merger interacting with an externally sourced magnetic field. We here describe the particular formulation of the d equations employed in our simulations. We begin with a brief review the $3+1$ decomposition og Genera Relativity followed by a discussion of the Cauchy problem for both Einstein and Maxwell equations.

\section{A. The $3+1$ decomposition}

In the Cauchy or $3+1$ formulation, the spacetime $\left(M, g_{a b}\right)(a, b=0,1,2,3)$ is foliated with spacelike hypersurfaces labeled by constant coordinate time $x^{0} \equiv t=$ const. The metric of these hypersurfaces is $\gamma_{i j}=g_{i j}$ $(i, j=1,2,3)$. The normal vector to the hypersurfaces is $n_{a} \equiv-\nabla_{a} t /\left\|\nabla_{a} t\right\|$, and coordinates defined on neighboring hypersurfaces can be related through the lapse function, $\alpha$, and shift vector, $\beta^{i}$. With these definitions, the spacetime line element can be expressed as

$$
\begin{aligned}
\mathrm{d} s^{2} & =g_{a b} \mathrm{~d} x^{a} \mathrm{~d} x^{b} \\
& =-\alpha^{2} \mathrm{~d} t^{2}+\gamma_{i j}\left(\mathrm{~d} x^{i}+\beta^{i} \mathrm{~d} t\right)\left(\mathrm{d} x^{j}+\beta^{j} \mathrm{~d} t\right)
\end{aligned}
$$

while the normal vector/covector is given explicity by

$$
n^{a}=\frac{1}{\alpha}\left(1,-\beta^{i}\right), n_{a}=(-\alpha, 0) .
$$

Indices on spacetime quantities are raised and lowered with the 4-metric, $g_{a b}$, and its inverse, while the 3 -metric $\gamma_{i j}$ and its inverse are used to raise and lower indices on spatial quantities. The following simple expressions relate the $3+1$ basic variables $\left\{\gamma_{i j}, \alpha, \beta^{i}\right\}$ with the fourdimensional metric $\left\{g_{a b}\right\}$ by

$$
\gamma_{i j}=g_{i j}, \quad \alpha=\sqrt{-1 / g^{00}}, \quad \beta^{i}=\gamma^{i j} g_{0 j} .
$$

In what follows we will make use of both sets of variables.

\section{B. Einstein equations}

The Einstein equations in the Generalized Harmonic formulation [12, 13] (GH) can be written as a system of ten nonlinear partial differential equations for the spacetime metric $g_{a b}$.

$$
\begin{aligned}
& g^{c d} \partial_{c d} g_{a b}+\partial_{a} H_{b}+\partial_{b} H_{a}=-16 \pi\left(T_{a b}-\frac{T}{2} g_{a b}\right) \\
& +2 \Gamma_{c a b} H^{c}+2 g^{c d} g^{e f}\left(\partial_{e} g_{a c} \partial_{f} g_{b d}-\Gamma_{a c e} \Gamma_{b d f}\right) \cdot(4)
\end{aligned}
$$

where the coordinates $x^{a}$ can be chosen to satisfy the generalized harmonic condition

$$
\nabla^{c} \nabla_{c} x^{a}=-g^{b c} \Gamma_{b c}^{a}=H^{a},
$$

for some arbitrary functions $H_{a}$. One possibility for determining these functions, which we adopt here, employs the original harmonic condition $H_{i}=0$ (i.e. for the spatial components), while a damped wave equation for the time one [14]:

$$
\nabla^{c} \nabla_{c} H_{t}=-\xi_{1} \frac{\alpha-1}{\alpha^{n}}+\xi_{2} n^{c} \partial_{c} H_{t} .
$$

Since there is no coupling between the principal part of the Einstein equations (4) with the generalized harmonic condition (6), the full system of equations (Einstein equations + gauge condition) is trivially hyperbolic, determined by wave-like equations with non-linear source terms.

A reduction to first order of the evolution system (ie, the Einstein equations (4) with the generalized harmonic condition (6) ) can be achieved by introducing new independent variables related to the time and space derivatives of the fields

$$
\begin{aligned}
Q_{a b} & \equiv-n^{c} \partial_{c} g_{a b}, \quad D_{i a b} \equiv \partial_{i} g_{a b} \\
G & \equiv-n^{c} \partial_{c} H_{t}, \quad G_{i} \equiv \partial_{i} H_{t}
\end{aligned}
$$

With these definitions we can write the evolution equations in our GH formalism in the following way [15, 16]

$$
\begin{aligned}
& \partial_{t} g_{a b}=\beta^{k} D_{k a b}-\alpha Q_{a b}, \\
& \partial_{t} Q_{a b}=\beta^{k} \partial_{k} Q_{a b}-\alpha \gamma^{i j} \partial_{i} D_{j a b} \\
& -\alpha \partial_{a} H_{b}-\alpha \partial_{b} H_{a}+2 \alpha \Gamma_{c a b} H^{c} \\
& +2 \alpha g^{c d}\left(\gamma^{i j} D_{i c a} D_{j d b}-Q_{c a} Q_{d b}-g^{e f} \Gamma_{a c e} \Gamma_{b d f}\right) \\
& -\frac{\alpha}{2} n^{c} n^{d} Q_{c d} Q_{a b}-\alpha \gamma^{i j} D_{i a b} Q_{j c} n^{c} \\
& -8 \pi \alpha\left(2 T_{a b}-g_{a b} T\right) \\
& -2 \sigma_{0} \alpha\left[n_{a} Z_{b}+n_{b} Z_{a}-g_{a b} n^{c} Z_{c}\right] \\
& +\sigma_{1} \beta^{i}\left(D_{i a b}-\partial_{i} g_{a b}\right) \text {, } \\
& \partial_{t} D_{i a b}=\beta^{k} \partial_{k} D_{i a b}-\alpha \partial_{i} Q_{a b} \\
& +\frac{\alpha}{2} n^{c} n^{d} D_{i c d} Q_{a b}+\alpha \gamma^{j k} n^{c} D_{i j c} D_{k a b} \\
& \text { - } \sigma_{1} \alpha\left(D_{i a b}-\partial_{i} g_{a b}\right), \\
& \partial_{t} H_{t}=\beta^{k} G_{k}-\alpha G \text {, } \\
& \partial_{t} G=\beta^{k} \partial_{k} G-\alpha \gamma^{i j} \partial_{i} G_{j} \\
& -\frac{\alpha}{2} G n^{c} n^{d} Q_{c d}-\alpha \gamma^{i j} G_{i} Q_{j c} n^{c}+\alpha \Gamma_{c} G^{c} \\
& +\sigma_{1} \beta^{i}\left(G_{i}-\partial_{i} H_{t}\right)-\xi_{1} \frac{\alpha-1}{\alpha^{n-1}}-\alpha \xi_{2} G, \\
& \partial_{t} G_{i}=\beta^{k} \partial_{k} G_{i}-\alpha \partial_{i} G \\
& +\frac{\alpha}{2} G n^{c} n^{d} D_{i c d}+\alpha \gamma^{j k} G_{k} n^{c} D_{i j c} \\
& \text { - } \sigma_{1} \alpha\left(G_{i}-\partial_{i} H_{t}\right) \text {. }
\end{aligned}
$$

This GH formulation includes a number of constraints that must be satisfied for consistency. Namely, two sets of first order constraints $\mathcal{C}_{i a b}, \mathcal{C}_{i j a b}$ and the four-vector $Z_{a}$ accounting for the physical energy and momentum constraints [15, 17],

$$
\mathcal{C}_{i a b} \equiv \partial_{i} g_{a b}-D_{i a b}=0
$$




$$
\begin{aligned}
\mathcal{C}_{i j a b} & \equiv \partial_{i} D_{j a b}-\partial_{j} D_{i a b}=0 \\
2 Z_{a} & \equiv-\Gamma_{a}-H_{a}\left(t, x^{i}\right)=0 .
\end{aligned}
$$

These constraints are controlled dynamically via the inclusion of a constraint damping mechanism 18], by adding certain terms proportional to these constraints (with free parameters $\sigma_{0}$ and $\sigma_{1}$ ) to the evolution equations.

\section{Maxwell equations}

The Maxwell equations can be written in covariant form as

$$
\begin{aligned}
\nabla_{b} F^{a b} & =4 \pi I^{a}, \\
\nabla_{b}{ }^{*} F^{a b} & =0
\end{aligned}
$$

where $F^{a b}$ is the Maxwell tensor of the electromagnetic field, ${ }^{*} F^{a b}$ is the Faraday tensor and $I^{a}$ is the electric current 4 -vector. Since $F^{a b}$ is antisymmetric, the divergence of equation (15) leads to the current conservation

$$
\nabla_{a} I^{a}=0 .
$$

When both the electric and magnetic susceptibility of the medium vanish, as in vacuum or in a highly ionized plasma, the Faraday tensor is simply the dual of the Maxwell one, that is

$$
{ }^{*} F^{a b}=\frac{1}{2} \epsilon^{a b c d} F_{c d}
$$

where $\epsilon^{a b c d}$ is the Levi-Civita pseudotensor of the spacetime, which can be written in terms of the 4-indices LeviCivita symbol $\eta^{a b c d}$ as

$$
\epsilon^{a b c d}=\frac{1}{\sqrt{g}} \eta^{a b c d} \quad \epsilon_{a b c d}=-\sqrt{g} \eta^{a b c d} .
$$

For intuitive and practical reasons, it is convenient to introduce and work with the electric and magnetic fields which are defined as

$$
E^{a}=F^{a b} n_{b} \quad, \quad B^{a}={ }^{*} F^{a b} n_{b} .
$$

The vectors $E^{a}$ and $B^{a}$ are the electric and magnetic fields measured by the normal observer and are purely spatial (ie, $E^{a} n_{a}=B^{a} n_{a}=0$ ). The Faraday tensor can then be re-expressed as,

$$
\begin{aligned}
F^{a b} & =n^{a} E^{b}-n^{b} E^{a}+\epsilon^{a b c d} B_{c} n_{d}, \\
{ }^{*} F^{a b} & =n^{a} B^{b}-n^{b} B^{a}-\epsilon^{a b c d} E_{c} n_{d} .
\end{aligned}
$$

We consider here an extended Maxwell system [19] defined as

$$
\begin{aligned}
\nabla_{a}\left(F^{a b}+g^{a b} \Psi\right) & =-4 \pi I^{b}-\sigma_{2} n^{b} \Psi, \\
\nabla_{a}\left({ }^{*} F^{a b}+g^{a b} \phi\right) & =-\sigma_{2} n^{b} \phi,
\end{aligned}
$$

which reduces to the standard Maxwell equations if $\Psi=0=\phi$. These extra scalar fields play the role "error fields" as they are tightly coupled to constraints violations. Moreover, their induced evolution equations, obtained by considering the divergence of the extended Maxwell equations, imply

$$
\begin{aligned}
& \nabla_{a} \nabla^{a} \Psi=-\nabla_{a}\left(\sigma_{2} n^{a} \Psi\right) \\
& \nabla_{a} \nabla^{a} \phi=-\nabla_{a}\left(\sigma_{2} n^{a} \phi\right),
\end{aligned}
$$

which are a generalization of the telegraph equation. Their structure ensures the constraints will propagate at the speed of light and will be damped within a timescale given by $\sigma_{2}^{-1}$. This strategy is similar to that defined in [20] for ideal MHD case.

The $3+1$ version of equations (23) 244), which are the ones implemented in our code, are

$$
\begin{aligned}
\left(\partial_{t}-\mathcal{L}_{\beta}\right) E^{i}- & \epsilon^{i j k} \nabla_{j}\left(\alpha B_{k}\right)+\alpha \gamma^{i j} \nabla_{j} \Psi= \\
& \alpha \operatorname{tr} K E^{i}-4 \pi \alpha J^{i} \\
\left(\partial_{t}-\mathcal{L}_{\beta}\right) B^{i}+ & \epsilon^{i j k} \nabla_{j}\left(\alpha E_{k}\right)+\alpha \gamma^{i j} \nabla_{j} \phi= \\
& \alpha \operatorname{tr} K B^{i} \\
\left(\partial_{t}-\mathcal{L}_{\beta}\right) \Psi+ & \alpha \nabla_{i} E^{i}=4 \pi \alpha q-\alpha \sigma_{2} \Psi \\
\left(\partial_{t}-\mathcal{L}_{\beta}\right) \phi+ & \alpha \nabla_{i} B^{i}=-\alpha \sigma_{2} \phi .
\end{aligned}
$$

with $\operatorname{tr} K$ the trace of the extrinsic curvature and where we have decomposed the current four-vector $I^{a}=q n^{a}+$ $J^{a}$, (with the current $J^{a}$ satisfying $J^{a} n_{a}=0$ ). Obviously the standard Maxwell equations in a curved background are recovered for $\Psi=\phi=0$. From equations (29) and (30) it follows that $\Psi$ and $\phi$ can be regarded as the normal-time integrals of the standard divergence constraints

$$
\nabla_{i} E^{i}=4 \pi q, \quad \nabla_{i} B^{i}=0 .
$$

Finally, just reming that the sources are coupled to the geometry by means of the stress energy tensor $T_{a b}$ and its trace $T \equiv g^{a b} T_{a b}$. For our case of interest, the stress energy tensor is given by,

$$
T_{a b}=\frac{1}{4 \pi}\left[F_{a}^{c} F_{b c}-\frac{1}{2} g_{a b} F^{c d} F_{c d}\right],
$$

which depends quadratically on the electric and magnetic fields.

\section{Implementation}

Our code implements both systems of equations where the constraints, as mentioned, are kept under control via different but related damping mechanisms: constraint damping for the Einstein equations [18] and the extended divergence cleaning for the Maxwell equations as explained in the previous section. We adopt boundary conditions defined via a combination of Sommerfeld and constraint preserving boundary conditions 21] for both 
systems. To this end, a characteristic decomposition of the Generalized Harmonic formalism eqns. (95 (13) is performed (at each hypersurface) with respect to the wave front propagation direction, given by a normalized spatial vector $\mathbf{m}$. This vector is orthogonal to a given boundary and belongs to and ordered orthonormal triad $\{\mathbf{l}, \mathbf{p}, \mathbf{m}\}$. This decomposition gives,

$$
\begin{aligned}
& g_{a b}, H_{t} \quad v=0 \\
& D_{l a b}, D_{p a b}, G_{l}, G_{p} \quad v=-\beta^{m} \\
& L_{a b}^{ \pm} \equiv Q_{a b}-\sigma_{2} g_{a b} \pm D_{m a b} \quad v=-\beta^{m} \pm \alpha \\
& L^{ \pm} \equiv G-\sigma_{2} H_{t} \pm G_{m} \quad v=-\beta^{m} \pm \alpha
\end{aligned}
$$

where the symbol $\{l, p, m\}$ replacing an index means the projection along the corresponding vector. The boundary conditions are applied only to the incoming modes (i.e., $\left\{L_{a b}^{-}, L^{-}\right\}$and $\left\{D_{l a b}, D_{p a b}, G_{l}, G_{p}\right\}$ if $\left.\beta^{m}>0\right)$ through their time derivatives. We here explain in detail the modes related to the metric modes, while the modes related to $H_{t}$ are treated in an analogous way. The Sommerfeld condition considered is of the type [21]

$$
\left(\partial_{t}+\partial_{r}+\frac{1}{r}\right)\left(g_{a b}-\eta_{a b}\right)=0
$$

where $\eta_{a b}$ is just the Minkowski metric. By taking a time derivative of this equation and rewriting it in terms of the incoming characteristic fields, the final form of the Sommerfeld conditions results:

$$
\partial_{t}\left[L_{a b}^{-}+\left(\sigma_{2}-\frac{1}{r}\right) g_{a b}\right]=0
$$

We apply boundary conditions for $\left\{D_{l a b}, D_{p a b}\right\}$ if $\beta^{m}>$ 0 . In this case we use the constraint preserving boundary conditions already given in [15], where the original time derivatives are corrected by the 4-index constraint defined in eqn. (14), namely

$$
\begin{aligned}
\partial_{t} D_{l a b} & =\partial_{t} D_{l a b}-\beta^{m} m^{i} P_{l}^{j} C_{i j a b}, \\
\partial_{t} D_{p a b} & =\partial_{t} D_{p a b}-\beta^{m} m^{i} P_{p}^{j} C_{i j a b},
\end{aligned}
$$

where $P_{a b} \equiv g_{a b}+n_{a} n_{b}-m_{a} m_{b}$ is the projection tensor on the boundary surface.

The incoming modes of the electromagnetic fields are defined via maximally dissipative conditions on the time derivatives, induced from the physical picture of a circumbinary disk present beyond the computational domain. The complete set of eigenvectors for the extended Maxwell equations (27]30), again with respect to ordered orthonormal triad $\{\mathbf{l}, \mathbf{p}, \mathbf{m}\}$, is given by the following list of eigenfields propagating with light speed $-\beta^{m} \pm \alpha$ :

$$
\begin{gathered}
E_{l} \pm B_{p} \quad, \quad E_{p} \mp B_{l}, \\
\Psi \pm E_{m}, \quad \phi \pm B_{m} .
\end{gathered}
$$

The boundary conditions in this case are just given by

$$
\begin{aligned}
& \partial_{t}\left(E_{l}-B_{p}\right)=0 \quad, \quad \partial_{t}\left(E_{p}+B_{l}\right)=0, \\
& \partial_{t}\left(\Psi-E_{m}\right)=0,
\end{aligned}
$$

We adopt Finite Difference techniques on a regular Cartesian grid to implement the overall system numerically. To ensure sufficient resolution is used in an efficient manner we employ adaptive mesh refinement techniques. To this end we adopt the HAD computational infrastructure that provides distributed Berger-Oliger style Adaptive Mesh Refinement (AMR) 22, 23] with full subcycling in time, together with a novel treatment of artificial boundaries [24]. The refinement regions are determined using truncation error estimation via a shadow hierarchy [25] and so they adapt dynamically as the evolution proceeds to guarantee a certain pre-specified tolerance is achieved. A fourth order spatial discretization satisfying a summation by parts rule together with a third order Runge-Kutta scheme for the time integration are used to help ensure stability of the numerical implementation [26]. We adopt a Courant parameter of $\lambda=0.2$ so that $\Delta t_{l}=0.2 \Delta x_{l}$ at each level= $l$; this ensures the implementation satisfies the CourantFriedrichs-Levy condition dictated by the principal part of the equations. However notice that the different damping terms (either at the gauge condition (6) or constraint damping) in turn add a further requirement of the form $\sigma \Delta t_{l} \simeq O(1)$. Since $\Delta t_{l}$ is considerably larger for the coarser grids (where the solution is obtained at large distances) we address this issue by making the different damping factors space dependent, and in particular

$$
\sigma_{i}= \begin{cases}\hat{\sigma}_{i} & r \leq r_{0} M \\ \hat{\sigma}_{i} e^{-\left(r-r_{o} M\right)^{2} /(10 M)^{2}} & r>r_{0} M\end{cases}
$$

$(i=0,1,2)$. For similar reasons we also consider a spatial dependence for $\xi_{i}$. While different options work well, for comparison purposes we adopt a similar strategy as in [27], thus

$$
\xi_{i} \rightarrow \hat{\xi}_{i} e^{-r^{2} /(40 M)^{2}} f_{i}
$$

with

$$
f_{1}=\left(2-e^{\frac{t-t^{*}}{18 M}}\right)\left(1-e^{\frac{\left(t-t^{*}\right)^{2}}{(15 M)^{2}}}\right) \quad, \quad f_{2}=1 ;
$$

with $t^{*}$ chosen to be $10 \mathrm{M}$ before the onset of merger. As mentioned, other options that "turn on" the gauge smoothly from a pure Harmonic condition to a generalized one as given by eqn. (6) work as well.

\section{PHYSICAL SETUP AND INITIAL DATA}

We consider both single and binary black hole simulations, immersed in an otherwise constant magnetic field like the one produced by a disk surrounding the black hole at large distances. As the electromagnetic fields interact with the curved spacetime, they will be dynamically distorted and eventually reach a quasi-stationary configuration. We adopt electromagnetic fields within astrophysically relevant values where their energy is several orders of magnitude smaller than the gravitational 
field energy and so they have a negligible influence on the black holes' dynamics. A simple estimate indicates this is the case for a large (and certainly astrophysical interesting) range of field strengths. To this end first we express the field in terms of units of $M^{-1}$

$$
B[1 / M]=1.2 \cdot 10^{-20}\left(\frac{M}{M_{\odot}}\right) B[G]
$$

so, fields up to $B_{o}=10^{18}\left(M_{\odot} / M\right) G$ will have energy densities $\leq 10^{-4}\left[M^{-2}\right]$. Additionally, within a sphere of radius $\simeq 100 M$, the total $\mathrm{EM}$ energy will be bounded by $\% 1 M$ if the field strength is $\leq 10^{16}\left(M_{\odot} / M\right) G$. Therefore, while we here adopt a field strength of $B_{o}=$ $10^{4}\left(M / 10^{8} M_{\odot}\right) \mathrm{G}$, our results are applicable to much stronger values since for fields up to $\simeq 10^{16}\left(M_{\odot} / M\right) G$ the effects of the electromagnetic fields on the geometry are negligible however the latter can have a profound effect on the former.

The analysis of the single black hole case will serve not only as a test for our numerical implementation, but also to understand the features of the initial transient, where the EM fields adapt to the geometry of the black hole spacetime, giving rise to an electric field and a deformation of the magnetic field (see also [28, 29]). As it was shown in [28], the quasi-stationary state is determined by Wald's solution [30] for a Kerr black hole immersed in a uniform magnetic field which is aligned with its spin. Let us consider the explicit form of Wald's solution, which describes a solution of the Maxwell equations in the test field case. We assume a Kerr black hole in Boyer-Lindquist (BL) coordinates, immersed in a uniform magnetic field [30].

$$
\begin{aligned}
d s^{2} & =-\left(1-\frac{2 M r}{\Sigma}\right) d t^{2}-\frac{4 M a r \sin ^{2} \theta}{\Sigma} d t d \phi \\
& +\left[\frac{\left(r^{2}+a^{2}\right)^{2}-\Delta a^{2} \sin ^{2} \theta}{\Sigma}\right] \sin ^{2} \theta d \phi^{2}+\frac{\Sigma}{\Delta} d r^{2}+\Sigma d \theta^{2}
\end{aligned}
$$

where $\Sigma=r^{2}+a^{2} \cos ^{2} \theta$ and $\Delta=r^{2}+a^{2}-2 M r$. On this background, the following tensor defines a solution of Maxwell equations:

$$
\begin{aligned}
F & =F_{10} \omega^{1} \wedge \omega^{0}+F_{13} \omega^{1} \wedge \omega^{3}+F_{20} \omega^{2} \wedge \omega^{0} \\
& +F_{23} \omega^{2} \wedge \omega^{3}
\end{aligned}
$$

where $\omega^{a} \wedge \omega^{b} \equiv \frac{1}{2}\left(\omega^{a} \otimes \omega^{b}-\omega^{b} \otimes \omega^{a}\right)$, with

$$
\begin{aligned}
& \omega^{0}=\left(\frac{\Delta}{\Sigma}\right)^{2}\left(d t-a \sin ^{2} \theta d \phi\right), \omega^{1}=\left(\frac{\Sigma}{\Delta}\right)^{2} d r \\
& \omega^{2}=\Sigma^{1 / 2} d \theta, \quad \omega^{3}=\frac{\sin \theta}{\Sigma^{1 / 2}}\left[\left(r^{2}+a^{2}\right) d \phi-a d t\right] \\
& F_{10}=B_{0}\left[\frac{a r \sin ^{2} \theta}{\Sigma}-\frac{M a\left(r^{2}-a^{2} \cos ^{2} \theta\right)\left(1+\cos ^{2} \theta\right)}{\Sigma^{2}}\right] \\
& F_{13}=B_{0} \frac{\Delta^{1 / 2} r \sin \theta}{\Sigma}, F_{20}=B_{0} \frac{\Delta^{1 / 2} a \sin \theta \cos \theta}{\Sigma} \\
& F_{23}=B_{0} \frac{\cos \theta}{\Sigma}\left[r^{2}+a^{2}-\frac{2 M r a^{2}\left(1+\cos ^{2} \theta\right)}{\Sigma}\right]
\end{aligned}
$$

Here $B_{0}$ is the magnitude of the magnetic field at large distances from the black hole. For convenience we write the solution's components in the standard coordinates $\{t, r, \theta, \phi\}$,

$$
\begin{aligned}
F & =F_{r t} \omega^{r} \wedge \omega^{t}+F_{r \phi} \omega^{r} \wedge \omega^{\phi}+F_{\theta t} \omega^{\theta} \wedge \omega^{t} \\
& +F_{\theta \phi} \omega^{\theta} \wedge \omega^{\phi}
\end{aligned}
$$

$F_{r t}=F_{10}-\frac{a \sin \theta}{\Delta^{1 / 2}} F_{13}, \quad F_{\theta t}=\Delta^{1 / 2} F_{20}-a \sin \theta F_{23}$,

$F_{r \phi}=-a \sin ^{2} \theta F_{10}+\frac{\left(r^{2}+a^{2}\right)}{\Delta^{1 / 2}} \sin \theta F_{13}$,

$F_{\theta \phi}=-a \Delta^{1 / 2} \sin ^{2} \theta F_{20}+\left(r^{2}+a^{2}\right) \sin \theta F_{23}$.

Lastly, since we employ excision techniques we prefer to adopt horizon penetrating coordinates and so transform this solution to Kerr-Schild coordinates by the transformation 31]

$$
\begin{aligned}
& d t \rightarrow d t-\frac{2 r}{\Delta} d r \\
& d \phi \rightarrow d \phi-\frac{a}{\Delta} d r
\end{aligned}
$$

The explicit expressions of the fields in these coordinates are lengthy but straightforward. To gain some insight on the solution however, the particular case of a nonspinning black hole suffices (setting $a=0$ ). The line element in the BL coordinates reduces to

$$
\begin{aligned}
d s^{2} & =-\left(1-\frac{2 M}{r}\right) d t^{2}+\left(\frac{r^{2}}{r^{2}-2 M r}\right) d r^{2} \\
& +r^{2} d \theta^{2}+r^{2} \sin ^{2} \theta d \phi^{2} .
\end{aligned}
$$

The electric field vanishes $\left(E^{i}=0\right)$ and the magnetic field components are given by

$$
B^{r}=B_{0} \alpha \cos \theta, B^{\theta}=-B_{0} \frac{\alpha \sin \theta}{r}, B^{\phi}=0 .
$$

The corresponding expressions in Kerr-Schild coordinates are:

$$
\begin{aligned}
& B^{r}=B_{0} \alpha \cos \theta, B^{\theta}=-B_{0} \frac{\alpha \sin \theta}{r}, B^{\phi}=0, \\
& E^{r}=E^{\theta}=0, E^{\phi}=\frac{2 B_{0} \alpha M}{r^{2}} .
\end{aligned}
$$

Notice that although the magnetic field has the same expression in both systems of coordinates, there appears a toroidal component in the electric field in these coordinates due to the non-vanishing shift vector.

To explore the effects of the merger dynamics on the electromagnetic field, we compare single spinning black hole with cases of equal-mass merging black holes. In all cases, the orbital plane of the evolution (or equatorial plane for the single $\mathrm{BH}$ ) is assumed to be aligned with that of the circumbinary disk. The magnetic field is defined as anchored in the disk; hence, its associated magnetic dipole is aligned with the orbital and spin angular momentum. 


\section{SINGLE BLACK HOLES; THE ASYMPTOTIC STATIONARY STATE}

For the single black hole case, we adopt a spinning black hole with a spin parameter given by $a=0.7 \mathrm{M}$ which is close to the spin expected for a merged black hole from an equal-mass, non-spinning binary system. We adopt this value for comparison with the binary black hole scenario presented in the next section, where the final spin can be calculated directly via simulations (for recent efforts in simulations and data analysis see e.g. 32 and references cited therein) or estimated by simple arguments as in [33]. In this simulation the geometry is kept fixed, in order to maintain the same initial choice of coordinates and be able to compare easily with Wald's (analytical) solution.

As mentioned, the initial magnetic field is described by a poloidal configuration constructed from the electromagnetic potential produced by a circular loop, whose radius is assumed to be larger than the region of interest [34]. We assume the disk lies at $10^{3} \mathrm{M}$, and for these distances the magnetic field is essentially constant within our computational domain, so we simply adopt $B^{i}=B_{o} \hat{z}$ and set the electric field initially to zero throughout. The magnetic field strength is $B_{o}=10^{4} \mathrm{G}$, which is consistent with possible values inferred in relevant astrophysical systems [35, 36].

Our numerical domain consists of a cubical region defined by $[-80 \mathrm{M}, 80 \mathrm{M}]^{3}$ with 80 points in the base grid. It employs an FMR configuration with 5 levels of refinement, each one covering half of the domain of the parent coarser level. Thus, the coarsest resolution employed is $\Delta x=2 M$ while the finest one is $\Delta x=0.125 M$. The damping parameter is set to be $\hat{\sigma}_{2}=1 M$.

The evolution shows an initial transient where the magnetic fields are deformed through the dynamics exhibit a twisting behavior around the spinning black hole as well as an induced electric field. After $t \simeq 80 \mathrm{M}$ the solution is clearly seen to evolve towards a quasistationary state determined by Wald's solution 30] for a Kerr black hole immersed in a uniform magnetic field which is aligned with its spin. This is illustrated in Fig. 1 which presents both the electric and magnetic field obtained at $t=200 M$ in the plane $y=0$ for $x>0$, the corresponding field from Wald's exact solution is shown for $x>0$. The apparent agreement along with a careful examination of the asymptotic solution indicates that, for a black hole immersed within an almost uniform magnetic field aligned with its spin, the final state is Wald's solution [28.

It is interesting to notice that the electric field configuration is equivalent to the one produced by a sphere with a surface density charge, inmersed in a external magnetic field. This is precisely the result expected within the membrane paradigm picture, which endows the event horizon with some physical properties (ie, temperature, current, density charge,etc.) [37]. From this point of view, the density charge distribution is thus responsible
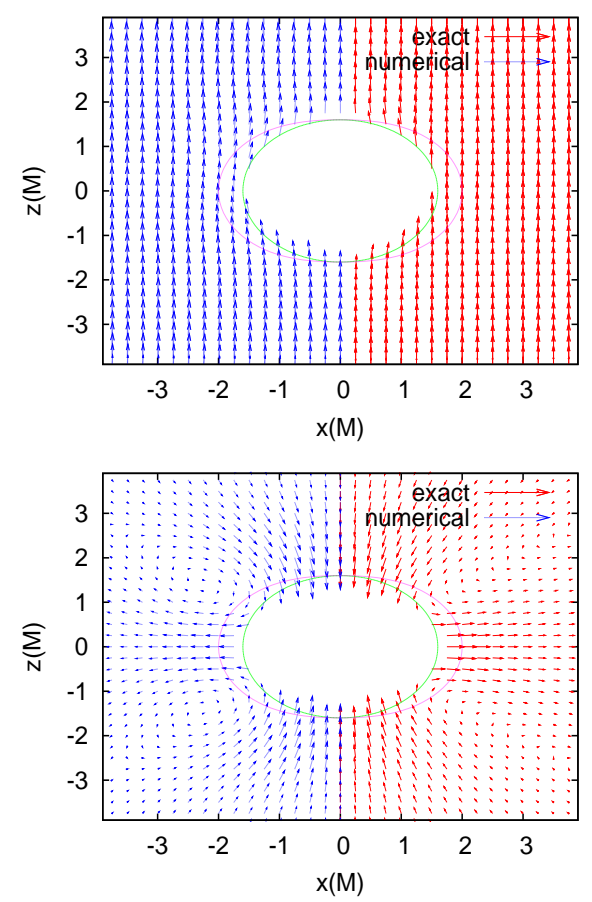

FIG. 1: Magnetic and electric field lines for $y=0, z \geq 0$ at $t=200 M$ for a single black hole, together with the apparent horizon (green) and the ergosphere (magenta). The regions $x<0$ display the numerical solution, while Wald's exact solution is shown in $x>0$.

for the electromagnetic field configuration observed.

\section{BINARY BLACK HOLES AND EFFECTS ON EM FIELDS}

We now turn our attention to the binary black hole case. As argued earlier the electromagnetic field considered (which is below estimated upper bounds in astrophysical scenarios) is not strong enough to affect the dynamics of the black holes, rather the black holes will affect the electromagnetic fields. Prior to merger, when the black holes are far from each other, the physical picture of the EM fields' behavior can be intuitively obtained from the knowledge of the black holes quasi-circular trajectories and the membrane paradigm point of view together with results from the previous section. Namely the black holes immersed in a uniform field affect the electromagnetic field's configuration in its local neighborhood, and the trajectories of the black holes cause a charge separation in the direction perpendicular to both the velocity and magnetic field, as in the Hall effect. As a result, the electromagnetic field induced by the binary motion is given by two dipoles in a quasi-adiabatical shrinking orbital behavior. As the merger stage approaches, the strong curvature and dynamics might affect the EM field's behavior more strongly and so we concen- 
trate on this stage. We adopt initial data such that the merger takes place after about one orbit. This initial data corresponds to quasi-equilibrium, equal-mass, nonspinning black holes constructed by the publicly available LORENE code 38]. The black holes have masses, given by $M_{s}=M / 2$, and are initially separated by $\approx 6 M$, lying beyond the approximate inner most stable circular orbit (ISCO) [33]. The initial magnetic field is chosen, as in the single black hole case, to be a poloidal configuration produced by a circular loop with large radius, so that $B^{i}=B_{o} \hat{z}$. The electric field is initially zero throughout the computational domain and the magnetic field strength adopted is $B_{o}=10^{4} \mathrm{G}$.

We adopt a cubical domain given by $[-106 M, 106 M]^{3}$ and employ an AMR configuration with 6 levels of refinement that adjust themselves dynamically to ensure that the solution's error is below a pre-determined threshold using a shadow hierarchy. The coarsest grid has 46 points, so the coarsest resolution is $\Delta x=4.6 M$ near the boundaries while the finest one is $\Delta x=0.072 M$ around the black holes. We adopt the following set of gauge parameters $\hat{\xi}_{1}=0.084 / M^{2}, \hat{\xi}_{2}=9 / M$ and $n=3$, while the damping parameters are $\hat{\sigma}_{0}=\hat{\sigma}_{1}=\hat{\sigma}_{2}=0.25 / M$. We monitor that the constraint remain well behaved through the evolutions; for instance fig. 2 displays the $L_{2}$ and the $L_{\infty}$ norms of the physical constraint $\|Z\|$ (14), defined as

$$
\|Z\| \equiv \sum_{a=0}^{3} Z_{a}^{2}
$$

Notice that besides a small increase at the time of the merger and at late times when the finest grid is automatically discarded and recreated a few times automatically as required by the shadow hierarchy, this constraint (as well as all others) remains under control during simulation.

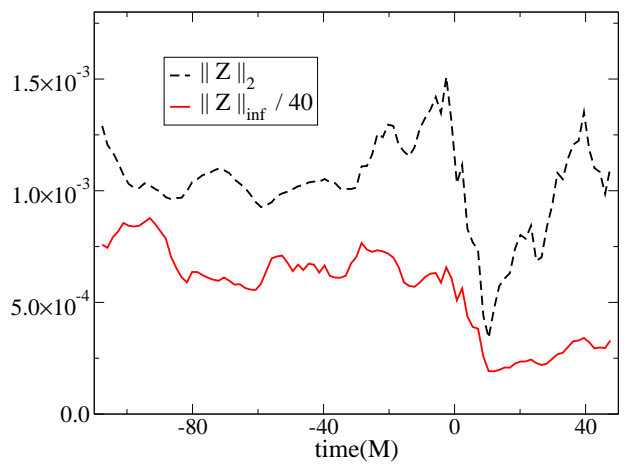

FIG. 2: $L_{2}$ and the $L_{\infty}$ norms of the physical constraint $\|Z\|$, which is kept under control through the evolution with a small increase at the merger. After it, the norms grow and oscillate for some period as the finest grid is detroyed and created a few times as required by the tolerance adopted at times $>38 M$ the finest is destroyed for the last time and not needed anymore.
A careful inspection of the dynamics described by the numerical simulation revealas that, except at late times after merger, the physical behavior results significantly different than the one corresponding to the single black hole case. Indeed the orbiting black holes modify both the geometry and the electromagnetic fields. As a result, the EM fields are stirred during the evolution, changing their configurations as it is displayed in Fig. 3.
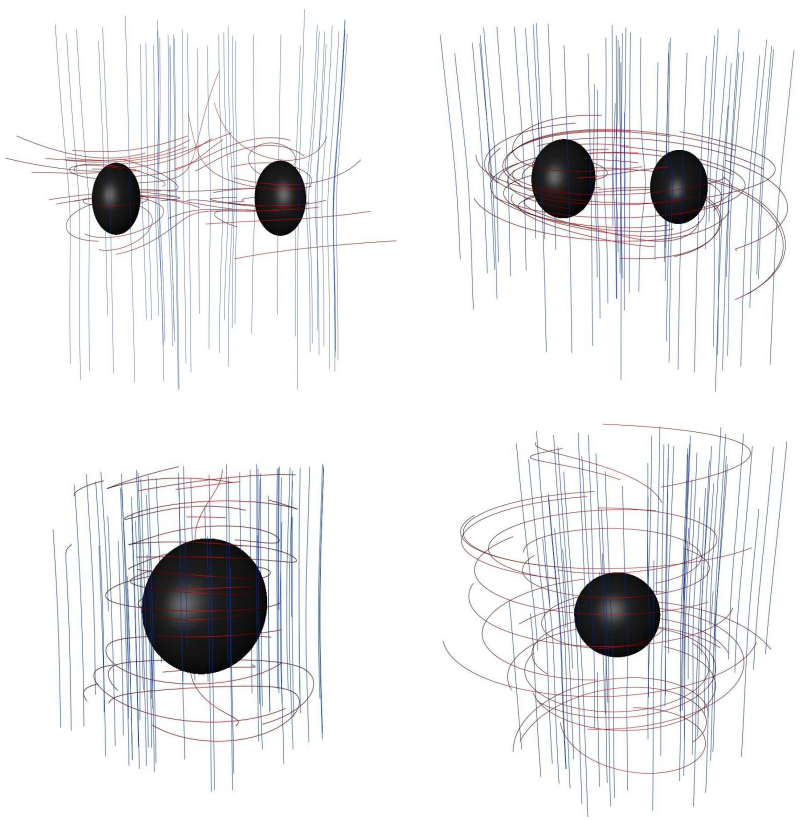

FIG. 3: Magnetic (mostly vertical) and electric field lines at different phases during the evolution employing different scales for visualization purposes. The figures illustrate different stages: early when the black holes are separated; near merger; shortly after they merge and at late times. The electric field lines are twisted around the black hole, while the magnetic lines slightly deform from ther initial configuration aligned with the $z$-axis.

To analyze the influence of the binary's dynamics on the electromagnetic field we monitor the (EM) NewmanPenrose radiative quantity $\Phi_{2}$, and correlate it with $\Psi_{4}$, which is the gravitational wave Newman-Penrose scalar. These scalars are computed by contracting the Maxwell and the Weyl tensor respectively, with a suitably defined null tetrad

$$
\Phi_{2}=F_{a b} n^{a} \bar{m}^{b} \quad, \quad \Psi_{4}=C_{a b c d} n^{a} \bar{m}^{b} n^{c} \bar{m}^{d} ;
$$

extracted at a sphere surface $\Sigma$ located in the wave-zone, far away from the sources. We also check that corrections required for possible gauge issues, as discussed in [39], are negligible in our present case. To understand the induced multipolar structure of these quantities we decompose them in terms of spin-weighted spherical harmonics, with spin weight $s=-1$ for $\Phi_{2}$ and $s=-2$ for $\Psi_{4}$ (since these are their respective spin-weights). These modes exhibit a very similar behavior, with the most relevant 

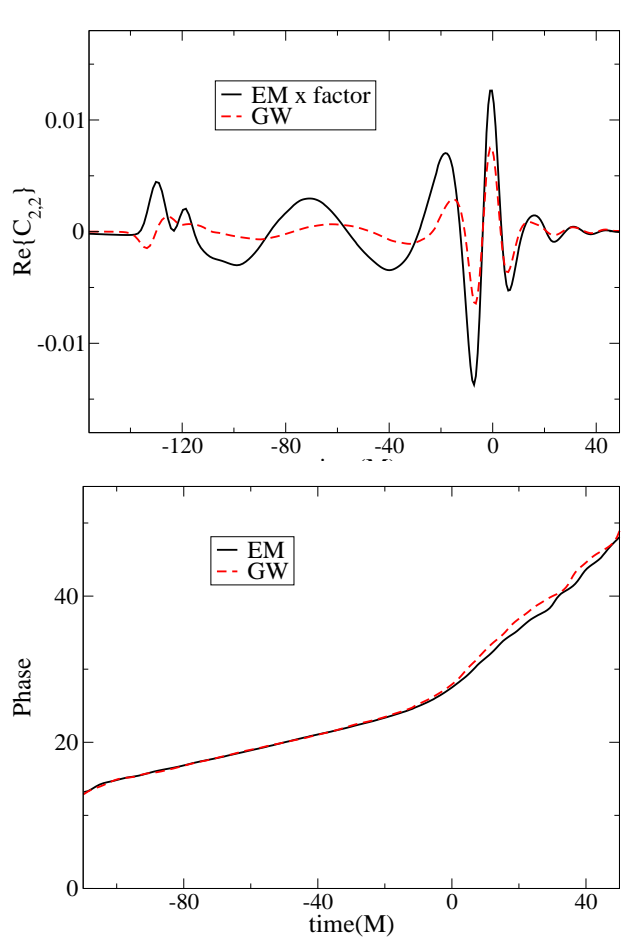

FIG. 4: The top figure corresponds to the $l=m=2$ modes for $r \Psi_{4}$ and $r \Phi_{2}$ extracted at $r=40 M$, reescaled properly with a factor $\approx 3 \times 10^{6}$ to fit in the same scale. The bottom figure illustrates the phase of these modes.

ones corresponding to the $l=2, m= \pm 2$ modes, which are plotted in fig. 4 (top). The maximum amplitudes of these waveforms correspond to the merger time which takes places after over one orbit. Note that since the magnetic field is anchored at the disk it does not decay with the distance from the binary, which obscures a clean interpretation from $\Phi_{2}$, displaying a non-vanishing $m=0$ mode at late times, when the stationary state is reached. The same happens with the decomposition of the radial component of the Poynting vector $S_{r}$ (radial from the origin), which shows non-radiative modes not related to the binary black hole dynamics. A closer inspection of the waveforms (bottom in fig. 4) reveals that the $l=$ $m=2$ mode of both the GW and the EM waves oscillate with the same frequency, indicating that both are mostly dominated by a quadrupolar structure resulting from the orbiting behavior.

The energy carried off by outgoing waves at infinity is another interesting quantity. The total energy flux per unit solid angle can be found directly from the NewmanPenrose scalars.

$$
\begin{aligned}
& F^{G W}=\frac{d E^{G W}}{d t d \Sigma}=\lim _{r \rightarrow \infty} \frac{r^{2}}{16 \pi}\left|\int_{\infty}^{t} \Psi_{4} d t^{\prime}\right|^{2}, \\
& F^{E M}=\frac{d E^{E M}}{d t d \Sigma}=\lim _{r \rightarrow \infty} \frac{r^{2}}{2 \pi}\left|\phi_{2}\right|^{2} .
\end{aligned}
$$

These quantities, integrated along the sphere surface $\Sigma$,

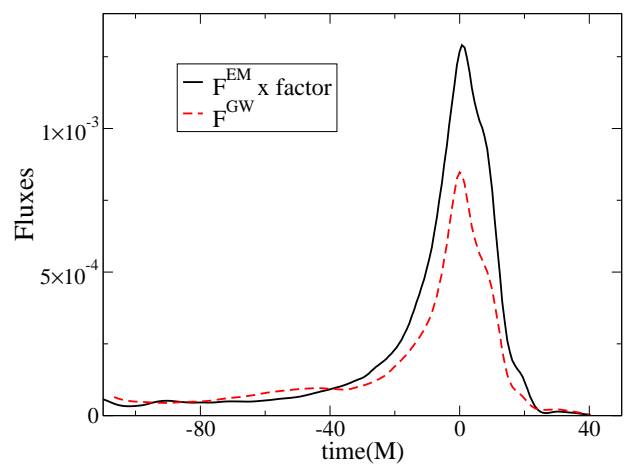

FIG. 5: Total flux of gravitational and electromagnetic energy corresponding to the binary black hole case (integrated over the sphere surface $\Sigma$ ). The (integrade) electromagnetic energy flux has been reescaled by a factor $\approx 10^{13}$ to make it appear clearly in the plot.

located at $R_{\Sigma}=30 M$ are shown in fig. 5 , both exhibiting a maximum at the time of the merger.

Furthermore, the electromagnetic energy density $\mathcal{E}_{E M} \equiv\left(E^{2}+B^{2}\right) / 2$, while not an invariant quantity in general relativity can be employed to get a sense of the energy variation in the EM field. As it is illustrated in Fig. 6. as the merger takes place, $\mathcal{E}_{E M}$ grows significantly during the merger and then diminishes as it approaches its asymptotic stage which is described by the scenario described in the single black hole case.

Summarizing, several distinct features in the EM fields behavior are seen during the evolution:

- After an initial transient dynamics, and well before the merger takes place, the electromagnetic fields display a pattern consistent with that produced by equal dipoles orbiting about each other, as it is exhibited in Fig. 7. This can be understood from the 'membrane paradigm' point of view [37], in which the horizon is endowed with a surface density of electrical charge. The quasi-circular trajectories of the black holes cause a charge separation in the direction perpendicular to both the velocity and magnetic field, inducing an electric and magnetic field in addition to the external one produced by the far away disk. To understand the main features observed a simple toy model could be employed. Such model is composed of four point charges (A,B,C,D) that orbit circularly. Assuming perfect conductivity of the horizon, each charge $q_{i}(i=A \ldots D)$ can be written as

$$
q_{A}=-\frac{r_{H}^{2}}{2 \pi}\left|v \times B_{o}\right|=-q_{B}=q_{C}=-q_{D},
$$

where $v$ is orbital velocity of the black holes, $B_{o}$ is the magnetic field imposed by circumbinary disk and $r_{H}$ is the radius of the apparent horizon. The orbital motion of each charge is written, in Cartesian coordinates, as:

$$
\overrightarrow{r_{i}^{\prime}}\left(t^{\prime}\right)=R_{i}(\cos \Omega t, \sin \Omega t, 0),
$$

where $R_{A}=R_{0}+r_{H}, R_{B}=R_{0}-r_{H}, R_{C}=-\left(R_{0}+\right.$ $\left.r_{H}\right), R_{D}=-\left(R_{0}-r_{H}\right)$, being $R_{0}$ the distance from 

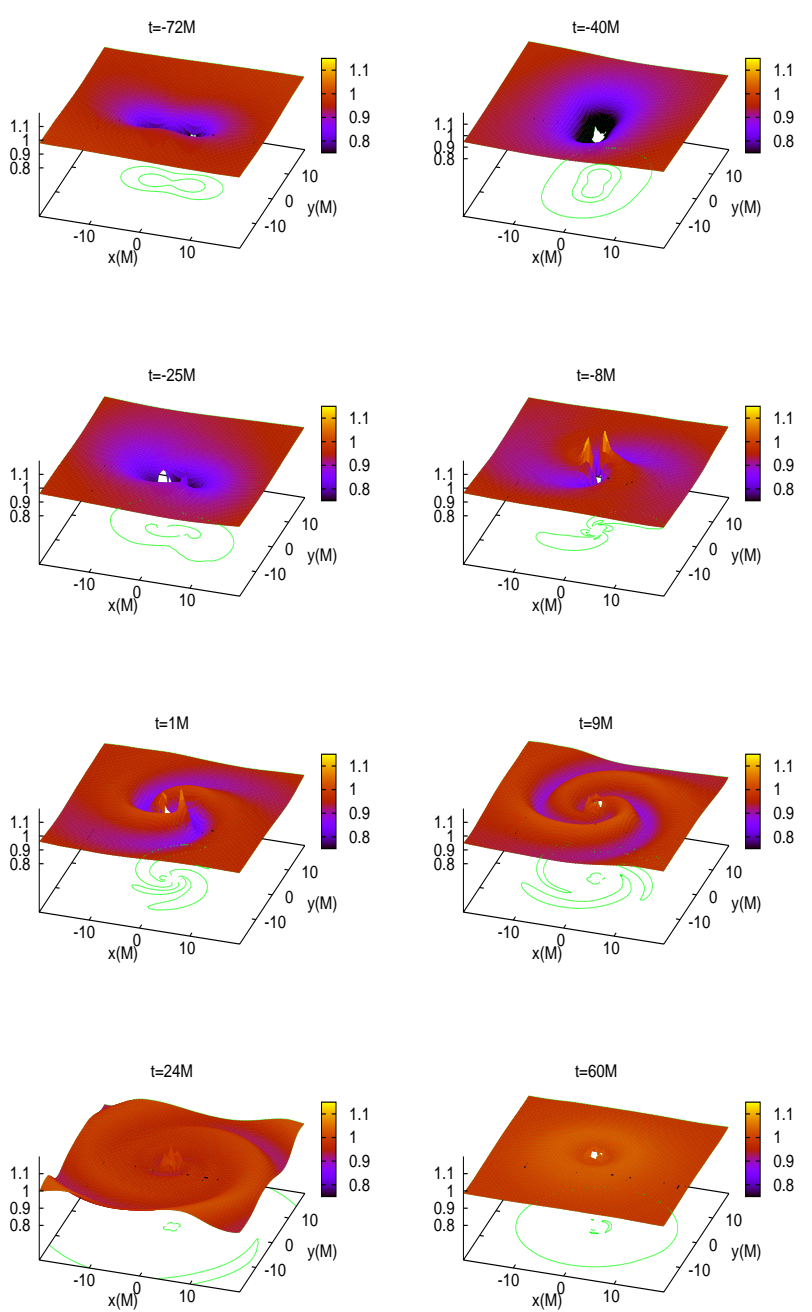

FIG. 6: Electromagnetic energy density (normalized to the values at the initial time, far distances) at the $z=0$ plane for the binary black hole case at different times of the evolution. Energy contour plots are shown from 1.2 to 0.8 at intervals of 0.1 .

either black hole to the origin (or center of mass). The field produced by this system can be computed by the following procedure. First, evaluate the Lienard-Wiechert potential of each particle and from it obtain its corresponding electric and magnetic field contribution. Next, sum up all contributions from the four particles to obtain the total electric and magnetic fields. Lastly, add the externalt magnetic field to the one produced by the charges. The final form of the fields at a given point involves implicit equations due to the different contributions depending on their respective retarded times. The intuitive picture however, can be derived easily by taking suitable limits. For instance, when the two black holes are well separated (ie, $R_{0}>>r_{H}$ ) the solution around each black hole mimics that of a dipole. At far distances
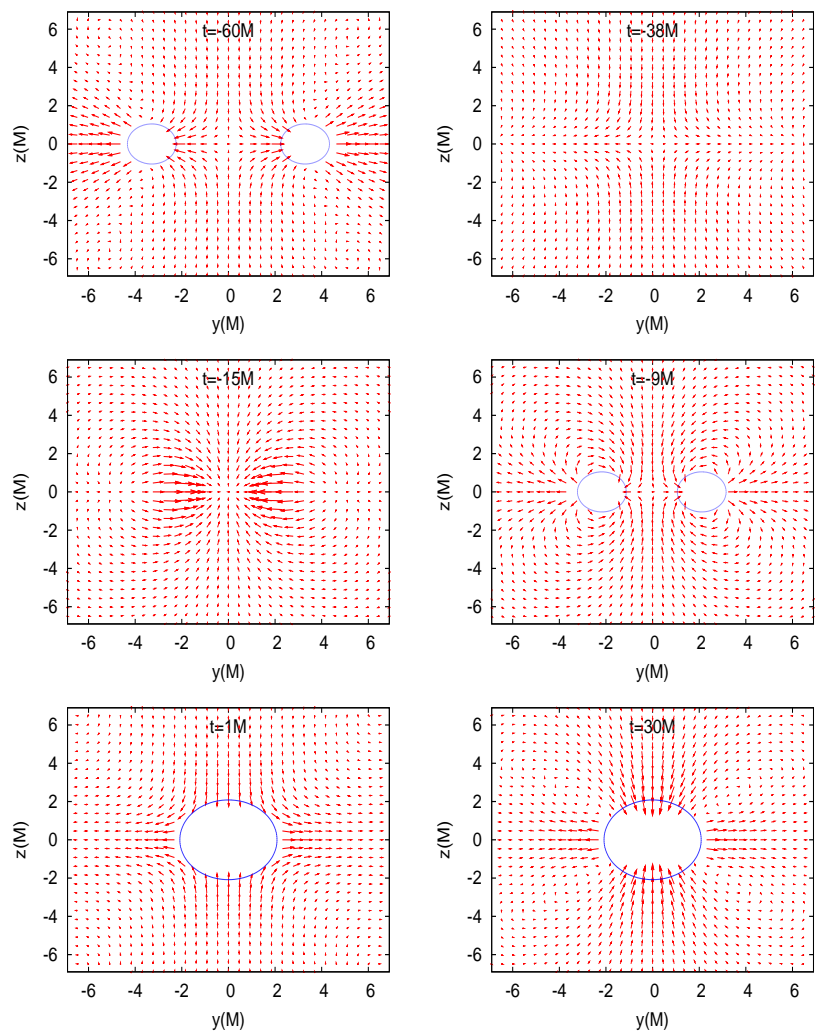

FIG. 7: Electric field lines at the plane $x=0$ for the binary black hole case at different times of the evolution. Notice that early on the field configuration is in agreement with the expected one from the membrane paradigm with two induced dipoles. At the black holes merge the configuration changes to a scenario consistent with that required in the BlandfordZnajek mechanism.

from the black holes, both the monopole and dipole contribution vanish and the system is described to leading order by a quadrupole. In this regime, the electric and magnetic fields at a point located at $\|\mathbf{r}\|>>R_{0}$, have the dependence

$\mathbf{E} \approx \frac{\Omega^{3}}{r} \cos \left(2 \Omega t+\delta_{E}\right), \quad \mathbf{B} \approx B_{o}+\frac{\Omega^{3}}{r} \cos \left(2 \Omega t+\delta_{B}(666)\right.$

where $\delta_{E}$ and $\delta_{B}$ are phases whose details are not important for the present argument. This simple form explains why $\phi_{2}$ and $\phi_{4}$ have the same frequency dependence $2 \Omega$.

- Near and through the merger epoch the fields' strengths increase and so does the flux of electromagnetic energy. Additionally the fields exhibit a configuration consistent with that required by the Blandford-Znajek mechanism, namely a (mainly) poloidal/toroidal magnetic/electric fields which could generate interesting energy ouput when interacting with a surrounding medium. As the merger approaches and take place, the field lines become stirred and twisted which, upon reconnection, could also release important amounts of energy, this scenario is beyond the scope of what we could study with 
our current setup.

- Finally, after the merger, the system can be understood again as a conducting sphere spinning in a external magnetic field. Its behavior can be understood by the results presented in the previous section.

\section{CONCLUSION}

We have analyzed the behavior of electromagnetic fields influenced by the dynamics of a binary black hole system. Our study illustrates several interesting aspects of such systems that emit not only gravitational waves, but can also radiate electromagnetically. Gravitational waves would be emitted through the different dynamical stages of the system -inspiral, merger and ringdownthrough a rather smooth manner with the peak occurring at the merger stage. Electromagnetic waves on the other hand, will be emitted through diverse processes driven by the interaction of the EM fields with surrounding plasma, gas or matter. In the current work we have studied the behavior of the EM fields and illustrated their "radiative" behavior as energy that can propagate outwards from the system as the black holes influence them. This energy will likely be absorbed and re-emitted by the surrounding plasma and would, in turn, be possible to observe. The orbiting behavior however, would leave its mark in a time variability naturally induced in the emission process.

Indeed, as we have illustrated here, the EM fields have a clearly discernible pattern tied to the dynamics of the system, making them possible tracers of the spacetime -in the electromagnetic sector- as these features would imprint particular characteristics in processes producing observable EM signals. In particular, in the pre-merger stages, the black hole dynamics induce EM flux oscillations with a period half that of the dominant GW signal produced by the system, i.e., a fourth of the orbital period, and a gradual enhancement of the energy in the electromagnetic field. This enhancement, together with a flux of electromagnetic energy would impact surrounding plasma in a stronger way than would be the case for a single stationary black hole as the latter would neither exhibit an enhancement, nor would it give rise to an outward flux of EM energy. Emissions in this later case require mechanisms like accretion or Blandford-Znajek to take place. Certainly, the same requirements will apply to binary black hole systems at late times as they give rise (generically) to a single spinning black hole. Furthermore, as the merger takes place fields are significantly twisted and stirred opening up the possibility of interesting emissions through magnetic reconnection. A study of such scenario requires a resistive treatment of the problem which is beyond the scope of our current work.

Perhaps even more exciting is the possibility of inducing a Blandford-Znajek analog for binaries as the merger proceeds. As we have seen, the system's dynamics induces a configuration consistent with the basic picture of this process and, by extracting rotational energy from the system, more powerful emissions could be expected. For this to take place however, an important conservative requirement should hold. Namely, an ergosphere must be present so that rotational energy can be extracted from the merging black holes. The best case scenario is for the ergosphere to form before the plunging phase begins so that the black holes can orbit and sufficient time is left for the extraction to occur. An estimate for when this takes places can be drawn from an "effective one body" approach, as described in the appendix, which indicates highly spinning configurations are required, at least in the particle limit, for an orbiting behavior to exist within the ergosphere.

Finally, and at a rather academically interesting level, it is interesting to ask what conditions would be required to extract so much energy that the end state is essentially a non-spinning black hole after the merger. For this to take place, the timescale of the BZ process $\left(\tau_{B Z}\right)$ should be comparable to that of the merger $\left(\tau_{M}\right)$. The latter typically lasts $\tau_{M} \simeq 50 M$, the former can be estimated as in [5] giving rise to

$$
\begin{aligned}
\tau_{B Z} & \simeq 5 \times 10^{8}\left(10^{15} G / B\right)^{2}\left(M_{\odot} / M\right) M_{\odot}, \\
& \simeq 10^{7}\left(10^{15} G / B\right)^{2}\left(M_{\odot} / M\right)^{2} \tau_{M}
\end{aligned}
$$

thus, for $B>10^{10} G$ both times result comparable for $\mathrm{BH}$ masses $\geq 10^{8} M_{\odot}$. Alternatively, one can estimate the amount of energy that could be extracted as a function of field strength within $\tau_{M}=50 M$. If $M_{p}$ is the final irreducible mass of the black hole after rotational energy has been extracted via the $\mathrm{BZ}$ mechanism, $M_{p}$ obeys

$$
\frac{M_{p}}{M-M_{p}} \simeq 10^{8}\left(10^{15} G / B\right)^{2}\left(M_{\odot} / M\right)^{2} .
$$

Thus, for $M=10^{9} M_{\odot}$ and $B=\left\{10^{7} / 10^{8}\right\} G$ about $\left\{10^{-4} / 10^{-2}\right\} M_{\odot} c^{2}$ (i.e. $\simeq\left\{10^{48} / 10^{50}\right\}$ ergs) is released in roughly a day.

While fields of these magnitudes might be unlikely, it is interesting that the strengths are not completely out of nature's ability to manifest. Beyond this possibility the impact of the dynamics on the electromagnetic fields is to induce a distinct variability as fields are dragged by the black holes. This suggests tantalizing prospects to detect pre-merger electromagnetic signals from systems detectable in the gravitational wave band. However, a complete description of the problem requires the incorporation of gas and radiation effects. Notwithstanding these missing -and important-ingredients, the main qualitative features - driven by the orbiting behavior of the black holes, whose inertia is many orders of magnitude above all else - would intuitively remain unaltered.

At a more speculative level, these combined signals could be exploited to shed light on possible observations 
to analyse alternative theories of gravity where photons and gravitons might propagate at different speeds or gravitational energy could propagate out of our possible 4-dimensional brane (for a recent discussion of some possibilities see [40, 41]).

As a final comment we stress that while our work is a step towards understanding possible emissions induced by binary black hole merger processes, we have only scratched the surface of possible phenomenology. For instance, within the current approach, scenarios with unequal masses and/or spins must be investigated and work is in progress to address them [42]. Still, a complete understanding of associated phenomena will require investigating, in particular, the interaction with a surrounding plasma and associated possible emission mechanisms. A preliminary related step in this direction has been considered in [9, 43] where the possibility of emission by a fossil gas in between the black holes has been considered.

Acknowledgments: We would like to thank M. Anderson, P. Chang, J. Frank, L. Rezzolla, S. Liebling, K. Menou, P. Moesta and D. Neilsen for stimulating discussions as well as to P. Grandclement for assistance with Lorene. This work was supported by the NSF grants PHY-0803629 and PHY-0653375 and also NSERC through a Discovery Grant. Computations were done at TeraGrid. LL acknowledges the Aspen Center for Physics for hospitality where this work was started. Research at Perimeter Institute is supported through Industry Canada and by the Province of Ontario through the Ministry of Research \& Innovation.

\section{APPENDIX}

The location for the innermost stable circular orbit, at the equator, in a black hole spacetime of mass $M$ and spin parameter $a$ is given by [44]

$$
Z_{1} \equiv 1+\left(1-\frac{a^{2}}{M^{2}}\right)^{1 / 3}\left[\left(1+\frac{a}{M}\right)^{1 / 3}+\left(1-\frac{a}{M}\right)^{1 / 3}\right]
$$

$$
\begin{aligned}
Z_{2} & \equiv\left(3 \frac{a^{2}}{M^{2}}+Z_{1}^{2}\right)^{1 / 2} \\
r_{I S C O} & =M\left(3+Z_{2} \mp\left[\left(3-Z_{1}\right)\left(3+Z_{1}+2 Z_{2}\right)\right]^{1 / 2}\right)
\end{aligned}
$$

The ergosphere, on the other hand, is located at

$$
r_{E R G O}=M+\sqrt{M^{2}-a^{2} \cos (\theta)^{2}}
$$

Thus, at the equatorial plane $r_{E R G O}=2 M$ while for prograde orbits $r_{I S C O}(a=0)=6 M$ and $r_{I S C O}(a=$ $M)=M$. Consequently, for sufficiently high spins $r_{E R G O}>r_{I S C O}$. This is illustrated in figure 8, where the critical value at which the two lines cross A related interesting point is that this argument bears relevance also to the generation of gravitational waves themselves. If the black holes orbit outside the isco but inside the ergosphere they could tap rotational energy and produced stronger emissions. All cases so far studied numerically lie below this critical value and so, if this simplistic model holds, it would indicate simulations have not yet probed the possibility of extraction of rotational energy. Therefore binary black hole systems could still potentially yield further interesting features in such regime.

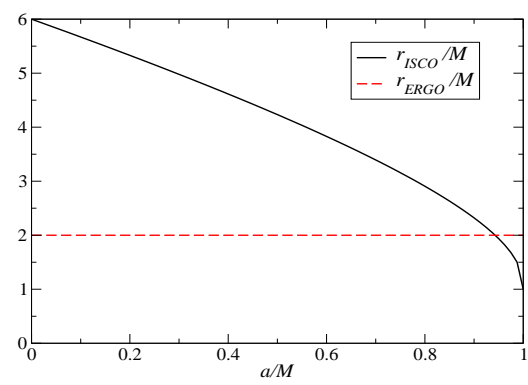

FIG. 8: ISCO and ergosphere radii vs $a / M$. For $a>0.943 M$ the ergosphere lies beyond the ISCO.
[1] J. Sylvestre, Astrophys. J. 591, 1152 (2003).

[2] C. W. Stubbs, Class. Quant. Grav. 25, 184033 (2008).

[3] R. Penrose, Riv. Nuovo Cim. 1, 252 (1969).

[4] R. D. Blandford and R. L. Znajek, Mon. Not. Roy. Astron. Soc. 179, 433 (1977).

[5] H. K. Lee, R. A. M. J. Wijers, and G. E. Brown, Physics Reports 325, 83 (2000), arXiv:astro-ph/9906213.

[6] M. C. Begelman, R. D. Blandford, and M. J. Rees, Nature (London) 287, 307 (1980).

[7] M. Milosavljevic and E. S. Phinney, Astrophys. J. 622, L93 (2005).

[8] Z. Haiman, B. Kocsis, and K. Menou (2009), 0904.1383.

[9] P. Chang, L. E. Strubbe, K. Menou, and E. Quataert (2009), 0906.0825.
[10] C. Palenzuela, M. Anderson, L. Lehner, S. L. Liebling, and D. Neilsen, Phys. Rev. Lett. 103, 081101 (2009), 0905.1121.

[11] J. R. van Meter et al. (2009), 0908.0023.

[12] H. Friedrich, Commun. Math. Phys. 100, 525 (1985).

[13] D. Garfinkle, Phys. Rev. D65, 044029 (2002), grqc/0110013.

[14] F. Pretorius, Phys. Rev. Lett. 95, 121101 (2005).

[15] L. Lindblom, M. A. Scheel, L. E. Kidder, R. Owen, and O. Rinne, Class. Quant. Grav. 23, S447 (2006), grqc/0512093.

[16] C. Palenzuela, I. Olabarrieta, L. Lehner, and S. Liebling, Phys. Rev. D75, 064005 (2007).

[17] C. Bona, T. Ledvinka, C. Palenzuela, and M. Žáček, 
Phys. Rev. D 67, 104005 (2003), arXiv:gr-qc/0302083.

[18] C. Gundlach et al., Class. Quant. Grav. 22, 3767 (2005).

[19] S. S. Komissarov, MNRAS 382, 995 (2007).

[20] A. Dedner et al., J. Comput. Phys. 175, 645 (2002).

[21] O. Rinne, L. Lindblom, and M. A. Scheel, Class. Quant. Grav. 24, 4054 (2007).

[22] Http://www.had.liu.edu/.

[23] S. L. Liebling, Phys. Rev. D66, 041703 (2002).

[24] L. Lehner, S. L. Liebling, and O. Reula, Class. Quant. Grav. 23, S421 (2006).

[25] F. Pretorius, Ph.D. thesis, The University of British Columbia (2002).

[26] M. Anderson et al., Phys. Rev. D77, 024006 (2008).

[27] M. A. Scheel et al., Phys. Rev. D79, 024003 (2009), 0810.1767.

[28] A. R. King, J. P. Lasota, and W. Kundt, Phys. Rev. D 12, 3037 (1975).

[29] S. S. Komissarov and J. C. McKinney, Mon. Not. Roy. Astron. Soc. Lett. 377, L49 (2007).

[30] R. M. Wald, Phys. Rev. D 10, 1680 (1974).

[31] S. S. Komissarov, MNRAS 350, 407 (2004).

[32] B. Aylott et al. (2009), 0901.4399.
[33] A. Buonanno, L. E. Kidder, and L. Lehner, Phys. Rev. D77, 026004 (2008).

[34] J. D. Jackson, Classical Electrodynamics (John Wiley \& Sons, New York, 1975).

[35] M. Massi and M. Kaufman, Astronomy and Astrophysics 477, 1 (2008).

[36] G. B. Field and R. D. Rogers, Astrophys. J. 403, 94 (1993).

[37] K. S. Thorne, R. H. Price, and D. A. MacDonald, Black holes: The membrane paradigm (1986).

[38] Http://www.lorene.obspm.fr/.

[39] L. Lehner and O. M. Moreschi, Phys. Rev. D76, 124040 (2007), 0706.1319.

[40] Z. Haiman et al. (2008), 0811.1920.

[41] J. S. Bloom et al. (2009), 0902.1527.

[42] P. Moesta, C. Palenzuela, L. Rezzolla, L. Lehner, S. Yoshida, and D. Pollney, in preparation (2009).

[43] A. I. MacFadyen and M. Milosavljević, Astrophys. J. 672, 83 (2008).

[44] J. M. Bardeen, W. H. Press, and S. A. Teukolsky, Astrophys. J. 178, 347 (1972). 